Research article

Open Access

\title{
Evaluation of Ki-67 proliferation and apoptotic index before, during and after neoadjuvant chemotherapy for primary breast cancer
}

\author{
Russell Burcombe ${ }^{1}$, George D Wilson², Mitch Dowsett ${ }^{3}$, Ifty Khan ${ }^{4}$, Paul I Richman 5 , \\ Frances Daley ${ }^{2}$, Simone Detre $^{3}$ and Andreas Makris 5
}

\author{
${ }^{1}$ Kent Oncology Centre, Maidstone Hospital, Hermitage Lane, Maidstone, Kent ME16 9QQ, UK \\ 2Department of Radiation Biology, William Beaumont Hospital, 3811 W. Thirteen Mile Road, 105-R1, Royal Oak, MI 48073, USA \\ 3Institute of Cancer Research, Royal Marsden Hospital, Fulham Road, London SW3 6JJ UK \\ ${ }^{4}$ The Royal London Hospital, Whitechapel, London E1 1BB UK \\ 5 Mount Vernon Hospital, Rickmansworth Road, Northwood, Middlesex HA6 2RN, UK \\ Corresponding author: Russell Burcombe, burcombe@nildram.co.uk
}

Received: 30 Dec 2005 Revisions requested: 6 Mar 2006 Revisions received: 16 May 2006 Accepted: 22 May 2006 Published: 21 Jun 2006

Breast Cancer Research 2006, 8:R31 (doi:10.1186/bcr1508)

This article is online at: http://breast-cancer-research.com/content/8/3/R31

(c) 2006 Burcombe et al.; licensee BioMed Central Ltd.

This is an open access article distributed under the terms of the Creative Commons Attribution License (http://creativecommons.org/licenses/by/2.0), which permits unrestricted use, distribution, and reproduction in any medium, provided the original work is properly cited.

\begin{abstract}
Introduction Biological markers that reliably predict clinical or pathological response to primary systemic therapy early during a course of chemotherapy may have considerable clinical potential. This study evaluated changes in Ki-67 labeling index and apoptotic index (Al) before, during, and after neoadjuvant anthracycline chemotherapy.
\end{abstract}

Methods Twenty-seven patients receiving neoadjuvant FEC (5fluorouracil, epirubicin, and cyclophosphamide) chemotherapy for operable breast cancer underwent repeat core biopsy after 21 days of treatment. Tissue from pre-treatment biopsy, day 21 and surgery was analysed for Ki-67 index and Al.

Results The objective clinical response rate was 56\%. Eight patients (31\%) achieved a pathological response by histological criteria; two patients had a near-complete pathological response. A reduction in Ki-67 index was observed in $68 \%$ of patients at day 21 and $72 \%$ at surgery; Ki-67 index increased between day 21 and surgery in 54\%. Al decreased in 50\% of tumours by day 21 , increased in $45 \%$ and was unchanged in one patient; $56 \%$ demonstrated rebound increases in $\mathrm{Al}$ by the time of surgery. Neither pre-treatment nor post-chemotherapy median Ki-67 index nor median Al at all three time points or relative changes at day 21 and surgery differed significantly between clinical or pathological responders and nonresponders. Clinical responders had lower median Ki-67 indices at day $21(11.4 \%$ versus $27.0 \%, p=0.02)$ and significantly greater percentage reductions in Ki-67 at day 21 than did nonresponders $(-50.6 \%$ versus $-5.3 \%, p=0.04)$. The median day$21 \mathrm{Ki}-67$ was higher in pathological responders $(30.3 \%$ versus $14.1 \%, p=0.046)$. A trend toward increased Al at day 21 in pathological responders was observed (5.30 versus $1.68, p=$ $0.12)$. Increased day-21 Al was a statistically significant predictor of pathological response $(p=0.049)$. A strong trend for predicting pathological response was seen with higher Ki-67 indices at day 21 and $\mathrm{Al}$ at surgery $(p=0.06$ and 0.06 , respectively).

Conclusion The clinical utility of early changes in biological marker expression during chemotherapy remains unclear. Until further prospectively validated evidence confirming the reliability of predictive markers is available, clinical decision-making should not be based upon individual biological tumour marker profiles.

\section{Introduction}

Primary breast carcinomas treated with neoadjuvant chemotherapy or primary systemic therapy (PST) provide an ideal model to evaluate the role of biological markers as predictive and prognostic factors. Many retrospective studies have identified patterns of biomarker expression before or after chemotherapy which have predictive or prognostic significance in

$\overline{\mathrm{Al}}=$ apoptotic index; $\mathrm{BSA}=$ bovine serum albumin; $\mathrm{cCR}=$ complete clinical response; $\mathrm{CR}=$ complete response; $\mathrm{DPX}=$ distrene plasticiser $\mathrm{xylene}$; $\mathrm{ER}=$ oestrogen receptor; $\mathrm{FEC}=5$-fluorouracil, epirubicin, and cyclophosphamide; $\mathrm{FNA}=$ fine needle aspiration; $\mathrm{LI}=$ labeling index (Ki-67 index); $\mathrm{PBS}=$ phosphate-buffered saline; $\mathrm{pCR}=$ complete pathological response; $\mathrm{PR}=$ partial response; $\mathrm{PST}=$ primary systemic therapy; $\mathrm{SD}=$ stable disease; TBS $=$ tris-buffered saline; TUNEL $=$ terminal transferase uridyl nick-end labeling; UICC = International Union Against Cancer. 
relation to different clinical endpoints. However, no single pretreatment marker that can accurately predict response to PST has been found to be of clinical utility to date. Despite high objective response rates to PST, a small proportion of patients will fail to respond or will progress during primary chemotherapy. The early identification of non-responders may spare these patients the unnecessary toxicity of ineffective chemotherapy and allow them to be offered alternative treatment strategies or non-cross-resistant regimens. Biological markers that can reliably predict clinical or pathological response early during a course of treatment therefore have considerable clinical potential.

In randomised clinical trials, PST confers equivalent survival and increased breast conservation rates compared with primary surgery and adjuvant cytotoxic chemotherapy $[1,2]$. Complete pathological response ( $\mathrm{pCR}$ ) is a strong prognostic indicator for prolonged disease-free and overall survival [3]. Patients achieving a complete clinical response (cCR) also have a statistically superior disease-free and overall survival advantage over clinical non-responders [3,4]. It should be acknowledged that in the smaller of these two studies [4], patients received some chemotherapy post-operatively. Clinical response is frequently used as a surrogate intermediate endpoint for predicting disease-free survival and outcome after primary chemotherapy; PCR is a valid intermediate surrogate endpoint for predicting overall survival.

The ability to biopsy breast tumours in situ during primary chemotherapy provides a unique opportunity to evaluate molecular markers in the tumour before and during treatment and to relate these changes to both clinical and pathological response. Immunohistochemical analysis of tumour material from repeat biopsies during treatment may therefore help unravel the complex molecular mechanisms that ultimately determine clinical outcomes and thereby provide more useful and reliable intermediate predictive and prognostic factors.

The nuclear antigen Ki-67 is a proliferation marker expressed only in cycling cells. A strong correlation between S-phase fraction and Ki-67 index has been demonstrated [5-7]. Consequently, quantitative assessment of Ki-67 staining on paraffinembedded tumour sections provides an accurate estimate of the proliferation index of individual tumours. Cytotoxic chemotherapy induces programmed cell death by apoptosis. The percentage of apoptotic cells in tumour sections may be measured by labeling fragmented DNA breaks and calculating the apoptotic index (Al) using the TUNEL (terminal transferase uridyl nick-end labeling) assay [8].

In this study, Ki-67 and apoptosis were assessed on histological material before, during, and after PST for operable breast cancer to evaluate whether early changes in proliferation or apoptosis predict clinical or pathological response to treatment.

\section{Materials and methods Treatment protocol}

A series of 39 female patients with operable (T2-T4, NO or $\mathrm{N} 1, \mathrm{MO}$ ) invasive primary breast carcinoma were identified between May 1999 and July 2001. Patients with metastatic disease (M1) or inflammatory breast cancer (T4d) were excluded. Core biopsy of the primary tumour was performed at diagnosis and repeated on day 21 , immediately prior to the second cycle of chemotherapy. Six cycles of FEC chemotherapy (5-fluorouracil $600 \mathrm{mg} / \mathrm{m}^{2}$, epirubicin $60 \mathrm{mg} / \mathrm{m}^{2}$, and cyclophosphamide $600 \mathrm{mg} / \mathrm{m}^{2}$ ) were administered at 21 -day intervals. Bi-dimensional clinical tumour measurements were recorded before every treatment. Four patients developed disease progression by clinical criteria during chemotherapy and proceeded to immediate surgery. The remaining women underwent breast-conserving surgery or mastectomy at the surgeon's discretion approximately 1 month after the final cycle of chemotherapy. All patients who were treated by breast-conserving surgery received post-operative radiation to the residual breast (40 Gy in 15 daily fractions plus 10-Gy boost to tumour bed in five fractions; $n=12$ ) plus or minus lymph nodes (50 Gy in 25 fractions for a period of 5 weeks; $n$ $=2$ ). Post-mastectomy chest wall radiation was delivered to 13 of 15 patients ( 11 chest wall only, 2 chest wall and nodes). No patient received post-operative chemotherapy. Women with oestrogen receptor (ER)-positive tumours received 5 years of adjuvant tamoxifen ( $20 \mathrm{mg}$ daily) starting after surgery.

The study was approved by the Luton \& Dunstable NHS (National Health Service) Trust Ethics Committee and conducted in accordance with the Helsinki Declaration.

\section{Assessment of response}

\section{Clinical response}

Standard UICC (International Union Against Cancer) criteria were used to define objective clinical response [9]. Changes in the calculated product of bi-dimensional tumour measurements on two successive evaluations were recorded at each visit. Complete response (CR) was defined as no residual palpable abnormality, partial response (PR) as greater than $50 \%$ tumour shrinkage, stable disease (SD) as less than $50 \%$ tumour shrinkage or no change, and progressive disease as an increase of at least $25 \%$.

\section{Pathological response}

Although many different systems for grading pathological response have been proposed [10-15], no standard method for pathological assessment after chemotherapy has been adopted. A previously described simple scoring system that can be applied in clinical practice was therefore employed [16]. A consultant histopathologist (P.I. Richman) blinded to clinical outcome reviewed all paired biopsy and surgical specimens. We defined 'histological tumour response' by both (a) an apparent reduction in tumour cell/stroma ratio and (b) one or more chemotherapy-induced cytological changes (that is, 
Table 1

\begin{tabular}{|c|c|c|c|}
\hline & & $n$ & Percentage \\
\hline Age: median & & 51 years & \\
\hline Age: range & & $29-65$ & \\
\hline \multirow[t]{3}{*}{ Menstrual status } & Pre & 14 & 52 \\
\hline & Peri & 6 & 22 \\
\hline & Post & 7 & 26 \\
\hline \multirow[t]{3}{*}{ Clinical TNM stage at diagnosis } & $\mathrm{T} 2$ & 12 & 44 \\
\hline & Т3 & 13 & 48 \\
\hline & $\mathrm{T} 4$ & 2 & 7 \\
\hline \multirow[t]{2}{*}{ Clinical node status } & No & 17 & 63 \\
\hline & $\mathrm{N} 1$ & 10 & 37 \\
\hline \multirow[t]{2}{*}{ Breast } & Right & 15 & 56 \\
\hline & Left & 12 & 44 \\
\hline \multirow[t]{2}{*}{ Breast surgery } & Wide excision & 12 & 44 \\
\hline & Mastectomy & 15 & 56 \\
\hline \multirow[t]{3}{*}{ Postoperative radiotherapy } & Nil & 2 & 7 \\
\hline & Breast & 12 & 44 \\
\hline & Chest wall & 13 & 48 \\
\hline \multirow[t]{2}{*}{ ER status (biopsy) } & ER-positive & 15 & 56 \\
\hline & ER-negative & 12 & 44 \\
\hline \multirow[t]{2}{*}{ HER-2 status (biopsy) } & HER-2-positive & 11 & 41 \\
\hline & HER-2-negative & 16 & 59 \\
\hline \multirow[t]{4}{*}{ Tumour grade } & Unknown* & 5 & 19 \\
\hline & G1 & 2 & 7 \\
\hline & G2 & 9 & 33 \\
\hline & G3 & 11 & 41 \\
\hline \multirow[t]{4}{*}{ Pathological T stage } & $\mathrm{pCR}$ & 0 & 0 \\
\hline & pT1 & 8 & 30 \\
\hline & pT2 & 17 & 63 \\
\hline & pT3 & 2 & 7 \\
\hline \multirow[t]{3}{*}{ Pathological N stage } & $\mathrm{pNO}$ & 11 & 41 \\
\hline & $\mathrm{pN} 1$ & 12 & 44 \\
\hline & $\mathrm{pNX}$ & 4 & 15 \\
\hline
\end{tabular}

*Grading not possible due to chemotherapy artefact. ER, oestrogen receptor; HER-2, human epidermal growth factor 2; TNM, tumour, node, metastasis.

enlarged cells with finely vacuolated cytoplasm, an enlarged vesicular nucleus with a prominent single eosinophilic nucleolus, or an enlarged hyperchromatic dense nucleus with an irregular outline). The following classification was used to score surgical specimens for pathological response: $C R$, no residual invasive carcinoma; $\mathrm{PR}$, residual invasive carcinoma with histological tumour response; and SD, residual invasive carcinoma with no histological tumour response.

\section{Immunohistochemical technique}

Four-micrometer sections were dried overnight at $37^{\circ} \mathrm{C}$. Prior to antibody staining, the slides were pre-treated with micro- 
wave irradiation to unmask binding epitopes. After blocking of endogenous peroxidase activity with a $3 \%$ solution of hydrogen peroxide in methanol for 30 minutes, slides were immersed in $200 \mathrm{ml}$ of $10 \mathrm{mM}$ citric acid (pH 6.0) for 4 minutes on high power $(800 \mathrm{~W})$. After topping up of the buffer with distilled water, this step was repeated. The slides were then left to stand for 10 minutes in buffer at room temperature before being washed thoroughly in tap water. After three washes in tris-buffered saline (TBS), the slides were incubated with a 1:200 dilution of rabbit anti-Ki-67 polyclonal antibody (A0047; Dako UK Ltd., Cambridgeshire, UK) in TBS for 1-2 hours at room temperature. After three more washes in TBS, biotinylated goat anti-rabbit (Ki-67) in TBS was applied for 1 hour at room temperature. After an additional three washes, $A B C$ complex (K0355; Dako UK Ltd.) was added for 1 hour at room temperature. The staining was visualised by adding diaminobenzidine (DAB kit SK 4100; Vector Laboratories, Burlington, CA, USA) for 5 minutes at room temperature. The slides were washed well in tap water and counterstained with Mayers haematoxylin for 10 seconds to 1 minute and then dehydrated, cleared, and mounted in distrene plasticiser xylene (DPX). Positive and negative controls were performed with each batch of slides. Paired core biopsies and surgical specimens from the same patient were stained on the same run.

Apoptotic cells were visualised using a commercial end labeling (TUNEL) assay previously described [8]. Briefly, endogenous peroxidase activity was inactivated with $1 \%$ hydrogen peroxide in phosphate-buffered saline (PBS) $(\mathrm{pH} 7.4)$ for 10 minutes. Nuclei of tissue sections were stripped of proteins by incubation with $0.5 \%$ pepsin $(\mathrm{pH} 2.0)$ (Sigma Chemical Co, Poole, Dorset, UK) for 30 minutes at $37^{\circ} \mathrm{C}$. The sections were washed five times in distilled water to remove all traces of pepsin. Each section undergoing the TUNEL protocol was incubated for 5 minutes in Tris buffer $(\mathrm{pH} 7.6)$ and then for 1 hour at $37^{\circ} \mathrm{C}$ in $100 \mu$ of reaction mixture consisting of 15 units TdT FPLC pure (Pharmacia, Windsor, Berkshire, UK), $0.5 \mathrm{nmol}$ biotin-16-dUTP (Boehringer Mannheim, Mannheim, Germany), $5 \mathrm{mM}$ cobalt chloride, $0.2 \mathrm{M}$ sodium cacodylate, $25 \mathrm{mM}$ Tris $\mathrm{HCl}(\mathrm{pH} 6.6)$, and $0.25 \mathrm{mg} / \mathrm{ml}$ bovine serum albumin (BSA) dissolved in distilled water. After extensive washing in distilled water, the sections were incubated for 30 minutes at room temperature in 1:400 dilution of horseradish peroxidase conjugated to streptavidin (Dako UK Ltd.) in PBS supplemented with 1\% BSA and 0.5\% Tween 20. Colour was developed for 10 minutes using $0.05 \%$ diaminobenzidine plus $0.07 \%$ imidazole plus $0.1 \%$ hydrogen peroxide and further intensified in $0.5 \%$ copper sulphate with $0.9 \%$ sodium chloride for 1 minute. The sections were counterstained in Mayers haematoxylin, dehydrated, cleared in xylene, and mounted in DPX.

\section{Scoring methods}

Immunohistochemical scoring was performed without prior knowledge of the clinical response. Ki-67 score was counted on a minimum of 10 randomly selected $\times 40$ high-power fields containing representative sections of tumour and calculated as the percentage of positively stained cells to total cells. The Al was assessed by counting at least 3,000 malignant cells at $\times 400$ magnification. Stained apoptotic cells were recorded, and cells displaying classic apoptotic morphology but not staining were also incorporated in the Al. Non-staining apoptotic cells were recognised in the midst of cells with normal morphology by having either condensed, irregular nuclei frequently with a crescent-shaped appearance or fragmented nuclei within cells showing cytoplasmic withdrawal. Areas with extensive necrosis were avoided.

\section{Statistical methods}

Statistical analysis was carried out using JMP version 5.0 (SAS Institute Inc., Cary, NC, USA). Associations between ordinal variables were assessed using $\chi^{2}$ analyses or the Fisher exact test in the case of two-by-two variables. Analyses involving $\mathrm{Ki}-67$ and $\mathrm{Al}$ as continuous variables were investigated using analysis of variance. A logistic regression analysis was performed.

\section{Results \\ Day-21 biopsy}

Sufficient invasive carcinoma suitable for immunohistochemical analysis was present in 27 of the 39 day- 21 biopsies. The remaining 12 patients were excluded from the analysis: eight yielded no demonstrable invasive tumour on day-21 biopsy, two comprised high-grade DCIS (ductal carcinoma in situ) only (presumably due to geographical miss), and two contained tiny foci of invasive tumour deemed too small to reliably interpret immunohistochemical staining.

\section{Patient demographics}

Of the 27 evaluable patients, $52 \%$ were pre-menopausal. Most tumours were grade $2(33 \%)$ or $3(41 \%)$, stage T2 $(44 \%)$ or T3 $(42 \%)$, and clinically node-negative $(63 \%)$ before treatment. Fifty-six percent were ER-positive and $41 \%$ HER-2 (human epidermal growth factor-2)-positive. The patient characteristics are shown in Table 1.

\section{Response rates}

All 27 patients were evaluable for clinical response on completion of chemotherapy. Surgical blocks were retrieved for pathological scoring in 26 cases. The objective clinical response rate $(C R+P R)$ was $56 \%$ (15/27). Four patients $(15 \%)$ progressed by clinical criteria after two, two, four, and six cycles of chemotherapy, respectively, and proceeded to immediate surgery. The remaining eight patients $(30 \%)$ had clinically stable disease on completion. Eight patients (30\%) achieved a pathological response by histological criteria. There were no complete pathological responders, although two women had a 'near-pCR' with residual foci of invasive carcinoma measuring 1 and $2 \mathrm{~mm}$ in maximum dimension, respectively. 
Figure 1

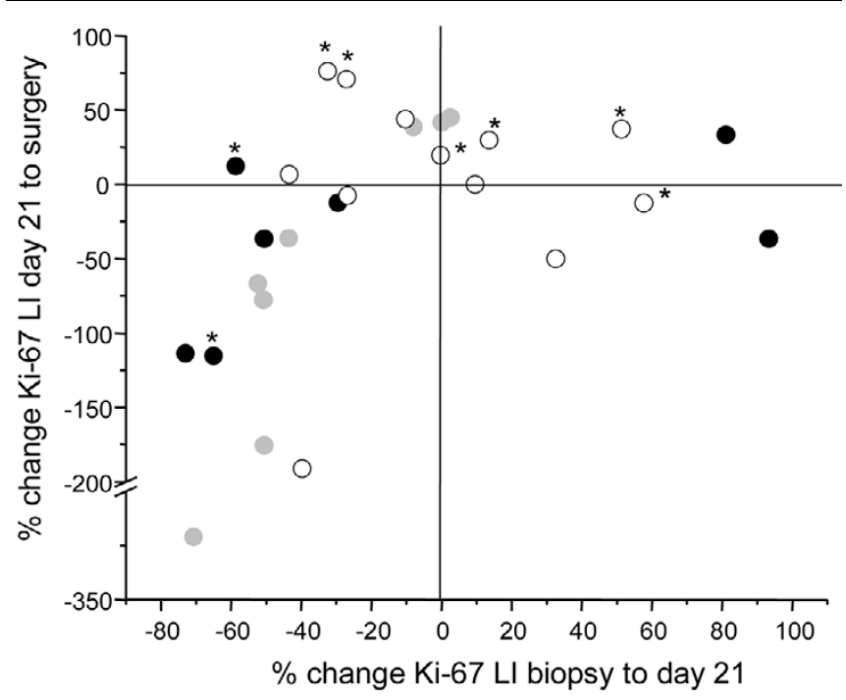

Changes in Ki-67 LI during treatment and clinical and pathological response. The data are expressed as \% change between initial biopsy and day 21 relative to the initial biopsy score (x-axis) versus \% change between day 21 and surgery relative to the day 21 index. (0) represents patients with a complete clinical response, $(\bullet)$ with a partial response and $(\bigcirc)$ represents no response. The asterisks represent those patients who achieved a pathological response.

\section{Biological markers before, during, and after chemotherapy}

The median and range of $\mathrm{Ki}-67$ indices before chemotherapy, at day 21 , and after treatment were $27.9 \%(4.1 \%-43.9 \%)$, $17.3 \%(4.1 \%-44.8 \%)$, and $21.7 \%$ (2.4\%-50.4\%), respectively. The apoptotic indices at baseline, day 21 , and surgery were $1.92 \%(0.23 \%-5.4 \%), 1.69 \%(0.33 \%-11.2 \%)$, and $2.19 \%(0.9 \%-4.9 \%)$, respectively. At each time point, there was a significant positive relationship between these two parameters: the correlation coefficients were 0.47 ( $p=$ $0.026), 0.65(p=0.0005)$, and $0.66(p=0.0014)$ in the biopsy, day-21 and surgery samples, respectively.

\section{Changes in biological markers during and after chemotherapy}

A reduction in $\mathrm{Ki}-67$ index from pre-treatment values was observed in 63\% (17/27) of patients at day 21 and 69\% (18/ 26 ) at surgery (Figure 1); there was no tumour material available for one patient at surgery. Eleven patients demonstrated sequential reductions in $\mathrm{Ki}-67 \mathrm{LI}$ throughout the two study periods, and four patients showed sequential increases during therapy. Four of the 17 tumours that showed a reduction in $\mathrm{LI}$ between biopsy and day 21 showed increases in proliferation between day 21 and surgery. Of the 10 tumours that showed no change or an increase in Ki-67 LI during the first 3 weeks of chemotherapy, half displayed a subsequent reduction between day 21 and surgery.
Figure 2

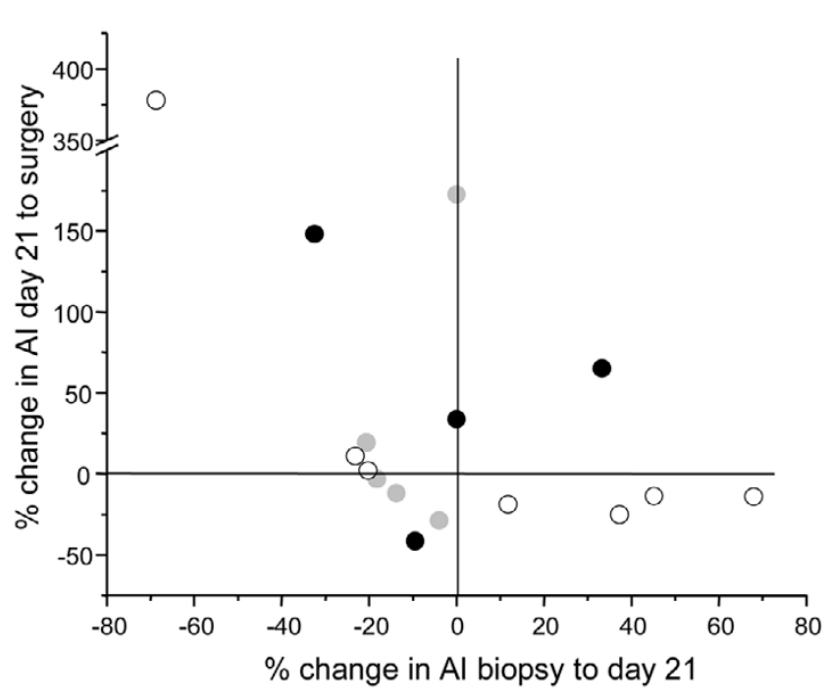

Changes in apoptotic LI during treatment and clinical and pathological response. The data are expressed as $\%$ change between initial biopsy and day 21 relative to the initial biopsy score ( $x$-axis) versus \% change between day 21 and surgery relative to the day 21 index. $(\bullet)$ represents patients with a complete clinical response, $(\boldsymbol{\bullet})$ with a partial response and $(\bigcirc)$ represents no response. The asterisks represent those patients who achieved a pathological response.

The Al was more difficult to assess in this material. There were seven instances in the day-21 biopsies and nine in the surgical material in which it was not possible to make a reliable measurement with the TUNEL assay. In those cases that were evaluable, there was a wide variation in percentage change in $\mathrm{Al}$ at day 21 compared with pre-treatment levels. Al decreased in $50 \%(10 / 20)$, increased in $45 \%(9 / 20)$, and was unchanged in one patient (Figure 2). Overall, between initial biopsy and surgery, a similar pattern was seen with eight (47\%) out of 17 patients, with successful staining showing a reduction in $\mathrm{Al}$. Between day 21 and surgery, the majority of tumours (10 of 18) increased in apoptotic activity (Figure 2). Unlike in the Ki$67 \mathrm{LI}$ data, there was no consistent pattern in apoptosis throughout treatment.

\section{Ki-67 and clinical and pathological response}

Neither pre-treatment nor post-chemotherapy median Ki-67 index differed significantly between clinical or pathological responders and non-responders. Clinical responders (CR+PR) had significantly lower median Ki-67 indices at day 21 than did non-responders (11.4\% versus $27.0 \%, p=0.02$ ). A similar trend for lower day-21 Ki-67 in patients achieving a $c C R$ was also recorded $(p=0.10)$. Clinical responders exhibited significantly greater percentage reductions in $\mathrm{Ki}-67$ at day 21 than did non-responders $(-50.6 \%$ versus $-5.3 \%, p=0.04)$. A decrease or no change in day-21 Ki-67 was observed in $80 \%(12 / 15)$ of clinical responders compared with 58\% (7/ 12) of non-responders (Figure 1). In the 11 patients who showed sequential reductions in Ki-67 throughout the study 
period, nine (82\%) achieved a clinical response $(p=0.019)$ (Figure 1).

Paradoxically, the median day-21 Ki-67 was higher in pathological responders $(30.3 \%$ versus $14.1 \%, p=0.046)$. There were no association between pathological response and changes in Ki-67 throughout the study period and no correlation between clinical and pathological responses (Figure 1).

\section{Al and clinical and pathological response}

Median Al at all three time points and relative changes at day 21 and surgery did not differ significantly between clinical and pathological responders or non-responders (Figure 2). However, there was a trend toward higher pre-treatment $\mathrm{Al}$ in pathological responders (2.72 versus $1.65, p=0.10)$. A nonsignificant trend toward increased apoptosis at day 21 in pathological responders was also observed ( 5.30 versus $1.68, p=$ 0.12 ). No pattern in the distribution of changes in day-21 Al emerges between clinical and pathological responders when the data are represented graphically.

\section{Biological characteristics of complete or 'near-complete' pathological responders}

The ability to predict $\mathrm{pCR}$, arguably the most useful endpoint of all, could not be assessed in this cohort, because no patient achieved a pCR. However, two patients had only tiny foci of residual invasive carcinoma demonstrable after chemotherapy. Both these 'near-pCR' patients had a very high Al at operation (3.96 and 3.61), significantly greater than patients not achieving a 'near-pCR' $(p=0.04)$. One of these two patients was evaluable for day-21 Al; a large increase in $\mathrm{Al}$ was seen (5.3 versus 3.86) after the first cycle of chemotherapy. No clear trend in changes in Ki-67 during or after treatment was seen in the two patients with excellent pathological tumour regression.

\section{Logistic regression analysis for prediction of response by different modalities of assessment}

Logistic regression analyses were performed to establish which, if any, of the biological marker variables measured at different time points could predict response outcomes by clinical, radiological, or pathological criteria (Table 2). Increased Al at day 21 was a statistically significant predictor of pathological response $(p=0.049)$. Similarly, greater Ki-67 indices at day 21 and higher Al at surgery displayed a strong trend for predicting pathological response $(p=0.06$ and 0.06 , respectively). Reductions in $\mathrm{Ki}-67$ and $\mathrm{Al}$ at day 21 were strongly predictive of better clinical response by UICC category ( $p=0.01$ and 0.02 , respectively). No significant associations were observed between the various biological markers and clinical CR or radiological response assessed by mammography and/or ultrasound. Low baseline Al was associated with poor worst radiological response $(p=0.04)$.

\section{Discussion}

The prognostic significance of pre-treatment Ki-67 index in breast tumours varies. Intuitively, rapidly proliferating tumours confer a poor prognosis, and the majority of studies confirm this association [17-26]. In some series, breast tumours with a high proliferative index have a worse prognosis despite endocrine treatment $[27,28]$ or chemotherapy [29]. However, other authors report no significant difference in outcome after chemotherapy or hormone treatment in patients with rapidly proliferating tumours compared with those with more slowly growing tumours [30-33].

Changes in tumour cell proliferation before and after pre-operative treatment have also been evaluated. A reduction in $\mathrm{Ki}-67$ index has been demonstrated after chemotherapy [30,34,35], tamoxifen therapy $[31,36]$, and chemoendocrine therapy $[37,38]$. More recently, studies have focused on the evaluation of early changes in cell proliferation during treatment by analysing Ki-67 index in repeat tumour samples taken at varying intervals during chemotherapy. Two studies at the Royal Marsden Hospital (London, UK) performed on cytological material obtained from fine needle aspiration (FNA) during chemoendocrine treatment showed that reductions in Ki-67 proliferation index after 10, 14, or 21 days significantly predict clinical response $[37,38]$. However, Billgren and colleagues demonstrated that a decrease of more than $25 \%$ in proliferating fraction after the first course of chemotherapy significantly predicted a reduced risk of disease recurrence $(p=0.033)$ but showed no correlation with local objective response [39]. Multivariate analysis revealed that the decrease in proliferating fraction significantly added prognostic information to lymph node status. In a similar study, patients who responded to neoadjuvant chemotherapy and concurrent tamoxifen were found to be more likely to have a reduction in $\mathrm{Ki}-67$ ten days after chemotherapy than were non-responders [40]. Post-treatment Ki-67 index is also of prognostic importance: In a series of 42 patients treated with primary chemotherapy, high proliferative index in residual tumour was associated with a worse diseasefree survival [41].

In this study, there was no significant difference in baseline $\mathrm{Ki}$ 67 or Al between responders and non-responders assessed by clinical, pathological, or radiological criteria. Both pre-treatment and post-chemotherapy cell proliferation and apoptosis failed to predict response by any modality of assessment.

More than two thirds of tumours exhibited a decrease from baseline Ki-67 index at day 21 and at surgery. There was no significant difference in the magnitude of the decrease in Ki-67 from baseline to surgery between different groups. The degree of cell proliferation measured before or after chemotherapy was not able to discriminate clinical or pathological responders from non-responders. Clinical responders were more likely to exhibit a reduction in Ki-67 index after one cycle of chemotherapy. This group also displayed larger relative decreases in 
Logistic regression analysis showing significant associations for prediction of response by different modalities of assessment and response classifications

\begin{tabular}{|c|c|}
\hline Response variable & $p$ value \\
\hline \multicolumn{2}{|l|}{ Pathological response (R/NR) } \\
\hline Ki-67 D21 & 0.0616 \\
\hline Al D21 & 0.0497 \\
\hline AI Sx & 0.0620 \\
\hline \multicolumn{2}{|l|}{ Pathological CR } \\
\hline \multicolumn{2}{|l|}{ Not assessable } \\
\hline \multicolumn{2}{|l|}{ 'Near' pathological CR } \\
\hline No significant associations & - \\
\hline \multicolumn{2}{|c|}{ Clinical response (CR/PR/SD/PD) } \\
\hline [Path $T$ stage $T 1$ versus $T 3$ & $0.0028]$ \\
\hline Ki-67 D21 & 0.0097 \\
\hline Al D21 & 0.0224 \\
\hline \multicolumn{2}{|l|}{ Clinical response (CR/PR/NR) } \\
\hline [Path $T$ stage $T 1$ versus $T 3$ & $0.0066]$ \\
\hline Ki-67 D21 & 0.0326 \\
\hline Al D21 & 0.0224 \\
\hline \multicolumn{2}{|l|}{ Clinical response (R/NR) } \\
\hline Ki-67 D21 & 0.0323 \\
\hline \multicolumn{2}{|l|}{ Clinical CR } \\
\hline No significant associations & - \\
\hline Radiological response (R/NR) & - \\
\hline \multicolumn{2}{|l|}{ No significant associations } \\
\hline \multicolumn{2}{|c|}{ Mammographic response (CR/PR/SD/PD) } \\
\hline No significant associations & - \\
\hline \multicolumn{2}{|l|}{ USS response (CR/PR/SD/PD) } \\
\hline \multicolumn{2}{|l|}{ No significant associations } \\
\hline \multicolumn{2}{|c|}{ Worst radiological response (CR/PR/SD/PD) } \\
\hline Al biopsy & 0.0418 \\
\hline \multicolumn{2}{|c|}{ Worst radiological response (CR/PR/NR) } \\
\hline No significant associations & - \\
\hline
\end{tabular}
$\mathrm{Al}$, apoptotic index; $\mathrm{CR}$, complete response;D21, day-21; NR, non-responder; PD, progressive disease; PR, partial response; R, responder; SD, stable disease; USS, ultrasound scan.

cell proliferation after the first cycle of treatment (median 50.6, range -73.0 to 93.3) than did non-responders (median 5.3 , range -43.4 to 57.7$)(p=0.04)$. Paradoxically, Ki-67 expression at day 21 was greater in pathological responders compared with non-responders, despite the fact that the distribution of pre-treatment $\mathrm{Ki}-67 \mathrm{LI}$ was similar in both groups. This observation seems counterintuitive because tumour regression would be expected to be accompanied by a reduc- tion in cell proliferation. However, there was no association between clinical response and those patients who achieved a partial pathological response. These findings underline the uncertainty surrounding the optimum method of assessment of response in biomarker studies and raise concerns that one (or perhaps both) of the classifications of response used in this study may not be a reliable surrogate endpoint. 
More than half the patients showed an increase in measured cell proliferation between day 21 and surgery. In responding patients, the reduction in Ki-67 index after one cycle of treatment was not sustained and was often followed by a rebound increase in cell proliferation by the time of surgery (responders 26.8 , range 2.4 to 48.0 ; non-responders 18.9 , range 6.8 to 50.4).

The observed changes in proliferation during treatment may have implications for determining the optimum duration of neoadjuvant chemotherapy prior to surgery for operable primary breast cancer. Recently published randomised clinical trials suggest that the addition of sequential taxane chemotherapy after four cycles of anthracycline PST increases clinical and pathological response rates and translates into improved overall survival $[15,42]$. The rebound increases in cell proliferation noted after six cycles of anthracycline treatment in this study may partly explain the superior clinical results achieved when patients are switched to non-cross-resistant chemotherapy regimens midway through neoadjuvant treatment. This phenomenon warrants further investigation to establish whether changes in tumour cell kinetics during treatment can identify which patients are most likely to benefit from sequential chemotherapy schedules.

Wide variations in Al were seen both during and after chemotherapy. The non-significant trend toward increased $\mathrm{Al}$ in pathological responders at day 21 was confirmed by the logistic regression analysis showing that increased day-21 $\mathrm{Al}$ is a statistically significant predictor of pathological response. This observation suggests that tumours exhibiting high levels of cell death after one cycle of chemotherapy are more likely to achieve pathological regression. The high Al seen in the two near-pCR patients at operation indicates that increased apoptosis after chemotherapy may also predict which patients will have a good pathological response. Analysis of a larger cohort is required to explore this hypothesis further. The magnitude of changes in Al during treatment did not predict clinical, radiological, or pathological response to treatment.

The optimum time point for detecting early cell kinetic changes that may predict clinical and pathological outcomes is unknown. Other groups have repeated FNA cytology 10 days after chemoendocrine treatment $[37,38]$. Day 21 was arbitrarily chosen as a convenient time in this study, to coincide with patients' return to hospital for their second cycle of chemotherapy, although there are no convincing data that it is the most appropriate time to test biomarkers. It is possible that 21 days after chemotherapy is too late to observe the peaks of apoptotic response and suppression of proliferation induced by cytotoxic treatment; there may be earlier times when the biologic response to treatment is more critically related to therapeutic outcome. Indeed, there is some evidence that apoptotic response after chemotherapy lasts for several days only [4345]. Ideally, serial biopsies may help to chart the precise pat- tern of changes in biological markers during treatment; realistically, however, large studies of this type are impractical, because few patients are likely to agree to repeated invasive tumour biopsies.

It is important to recognise the potential limitations of this study. Like most published series in this field, the number of patients reported is small. The use of tumour biopsies to assess molecular marker expression before and after treatment has become increasingly widespread as the search for predictive markers for neoadjuvant chemotherapy continues. Critics initially argued that this approach was subject to sampling error and intra-tumour variability. However, the widely quoted validation study by Ellis and colleagues [35] demonstrated that core biopsies accurately reflect the expression of biological markers in whole tumour sections.

In addition to clinical response, a novel descriptive histological response analysis was used to grade pathological response in this study. Although this system has not been prospectively validated or proven to relate directly to survival, the strong body of evidence that $\mathrm{pCR}$ is a good prognostic indicator for long-term survival justifies its use. Unfortunately, the analysis was hampered by the absence of complete pathological responders in this small series, forcing the authors to adopt the more widely used assessment of clinical response as an endpoint.

\section{Conclusion}

In this small study, pre-treatment or post-chemotherapy median Ki-67 index, median Al at all three time points, and relative changes at day 21 and surgery did not differ significantly between clinical or pathological responders and non-responders. Clinical responders achieved significantly greater percentage reductions in Ki-67 and lower median Ki-67 indices at day 21 than did non-responders. Pathological responders displayed higher median day-21 Ki-67 expression. Increased day$21 \mathrm{Al}$ was a statistically significant predictor of pathological response. A strong trend for predicting pathological response was seen with higher $\mathrm{Ki}-67$ indices at day 21 and $\mathrm{Al}$ at surgery.

The clinical utility of early changes in biological marker expression during chemotherapy remains unclear. For the time being, clinical decision-making should not be based upon individual biological tumour marker profiles until further prospectively validated evidence confirming the reliability of predictive markers is available. In the meantime, large prospective clinical trials of neoadjuvant chemotherapy should include parallel biological marker studies to facilitate immunohistochemistry and microarray analysis on histological tissue taken at various time points before, during, and after neoadjuvant chemotherapy to continue the search for clinically useful predictive biomarkers.

\section{Competing interests}

The authors declare that they have no competing interests. 


\section{Authors' contributions}

RJB performed core biopsies, collected tissue samples, carried out immunohistochemical staining and scoring, and drafted the manuscript. GDW assisted with statistical analysis and helped write the manuscript. MD helped perform TUNEL assays and gave advice on manuscript content. IK performed statistical analyses. PIR scored samples for pathological response. FD assisted with immunohistochemical staining. SD performed TUNEL assays. AM conceived the study, participated in its design and coordination, and helped finalise the manuscript. All authors read and approved the final manuscript.

\section{Acknowledgements}

The Breast Cancer Research Trust, the Dowager Countess Eleanor Peel Trust, and the Peel Medical Research Trust generously supported this work but played no role in the design or conduct of the study.

\section{References}

1. Makris A, Powles TJ, Ashley SE, Chang J, Hickish T, Tidy VA, Nash $A G$, Ford HT: A reduction in the requirements for mastectomy in a randomized trial of neoadjuvant chemoendocrine therapy in primary breast cancer. Ann Oncol 1998, 9:1179-1184.

2. Fisher B, Brown A, Mamounas E, Wieand S, Robidoux A, Margolese RG, Cruz AB Jr, Fisher ER, Wickerham DL, Wolmark N, et al:: Effect of preoperative chemotherapy on local-regional disease in women with operable breast cancer: findings from National Surgical Adjuvant Breast and Bowel Project B-18. J Clin Oncol 1997, 15:2483-2493.

3. Fisher B, Bryant J, Wolmark N, Mamounas E, Brown A, Fisher ER, Wickerham DL, Begovic M, DeCillis A, Robidoux A, et al:: Effect of preoperative chemotherapy on the outcome of women with operable breast cancer. J Clin Oncol 1998, 16:2672-2685.

4. Cleator SJ, Makris A, Ashley SE, Lal R, Powles TJ: Good clinical response of breast cancers to neoadjuvant chemoendocrine therapy is associated with improved overall survival. Ann Oncol 2005, 16:267-272.

5. Gasparini G, Boracchi P, Verderio P, Bevilacqua P: Cell kinetics in human breast cancer: comparison between the prognostic value of the cytofluorimetric S-phase fraction and that of the antibodies to $\mathrm{Ki}-67$ and PCNA antigens detected by immunocytochemistry. Int J Cancer 1994, 57:822-829.

6. Dawson $A E$, Norton JA, Weinberg DS: Comparative assessment of proliferation and DNA content in breast carcinoma by image analysis and flow cytometry. Am J Pathol 1990, 136:1115-1124.

7. Vielh P, Chevillard S, Mosseri V, Donatini B, Magdelenat H: Ki67 index and S-phase fraction in human breast carcinomas. Comparison and correlations with prognostic factors. Am J Clin Pathol 1990, 94:681-686.

8. Mainwaring PN, Ellis PA, Detre S, Smith IE, Dowsett M: Comparison of in situ methods to assess DNA cleavage in apoptotic cells in patients with breast cancer. J Clin Pathol 1998, 51:34-37.

9. Hayward JL, Carbone PP, Heuson JC, Kumaoka S, Segaloff A, Rubens RD: Assessment of response to therapy in advanced breast cancer: a project of the Programme on Clinical Oncology of the International Union Against Cancer, Geneva, Switzerland. Cancer 1977, 39:1289-1294.

10. Sataloff DM, Mason BA, Prestipino AJ, Seinige UL, Lieber CP, Baloch Z: Pathologic response to induction chemotherapy in locally advanced carcinoma of the breast: a determinant of outcome. J Am Coll Surg 1995, 180:297-306.

11. Akashi-Tanaka $S$, Tsuda $H$, Fukuda $H$, Watanabe T, Fukutomi $T$ : Prognostic value of histopathological therapeutic effects and mitotic index in locally advanced breast cancers after neoadjuvant chemotherapy. Jpn J Clin Oncol 1996, 26:201-206.

12. Chevallier $B$, Roche H, Olivier JP, Chollet $P$, Hurteloup P: Inflammatory breast cancer. Pilot study of intensive induction chem- otherapy (FEC-HD) results in a high histologic response rate. Am J Clin Oncol 1993, 16:223-228.

13. Honkoop AH, Pinedo HM, De Jong JS, Verheul HM, Linn SC, Hoekman K, Wagstaff J, van Diest PJ: Effects of chemotherapy on pathologic and biologic characteristics of locally advanced breast cancer. Am J Clin Pathol 1997, 107:211-218.

14. Kuerer HM, Newman LA, Buzdar AU, Dhingra K, Hunt KK, Buchholz TA, Binkley SM, Strom EA, Ames FC, Ross MI, et al.: Pathologic tumor response in the breast following neoadjuvant chemotherapy predicts axillary lymph node status. Cancer $J$ Sci Am 1998, 4:230-236.

15. Smith IC, Heys SD, Hutcheon AW, Miller ID, Payne S, Gilbert FJ, Ah-See AK, Eremin O, Walker LG, Sarkar TK, et al:: Neoadjuvant chemotherapy in breast cancer: significantly enhanced response with docetaxel. J Clin Oncol 2002, 20:1456-1466.

16. Burcombe RJ, Makris A, Richman PI, Daley FM, Noble S, Pittam M Wright D, Allen SA, Dove J, Wilson GD: Evaluation of ER, PgR, HER-2 and Ki-67 as predictors of response to neoadjuvant anthracycline chemotherapy for operable breast cancer. $\mathrm{Br} J$ Cancer 2005, 92:147-155.

17. Brown RW, Allred CD, Clark GM, Osborne CK, Hilsenbeck SG: Prognostic value of Ki-67 compared to S-phase fraction in axillary node-negative breast cancer. Clin Cancer Res 1996, 2:585-592.

18. Gaglia P, Bernardi A, Venesio T, Caldarola B, Lauro D, Cappa AP, Calderini $P$, Liscia DS: Cell proliferation of breast cancer evaluated by anti-BrdU and anti-Ki-67 antibodies: its prognostic value on short-term recurrences. Eur J Cancer 1993, 29A:1509-1513.

19. Gottardi O, Tabiadon D, Scanzi F, Bono A, Majno M, Ferrari M, Colombo P: Clinical and prognostic usefulness of Ki67 determination in breast carcinoma. Pathologica 1992, 84:15-22.

20. Lee AK, Loda M, Mackarem G, Bosari S, DeLellis RA, Heatley GJ, Hughes K: Lymph node negative invasive breast carcinoma 1 centimeter or less in size (T1a, bNOMO): clinicopathologic features and outcome. Cancer 1997, 79:761-771.

21. Pierga JY, Leroyer A, Viehl P, Mosseri V, Chevillard S, Magdelenat $\mathrm{H}$ : Long term prognostic value of growth fraction determination by Ki-67 immunostaining in primary operable breast cancer. Breast Cancer Res Treat 1996, 37:57-64.

22. Pinder SE, Wencyk P, Sibbering DM, Bell JA, Elston CW, Nicholson R, Robertson JF, Blamey RW, Ellis IO: Assessment of the new proliferation marker MIB1 in breast carcinoma using image analysis: associations with other prognostic factors and survival. Br J Cancer 1995, 71:146-149.

23. Railo M, Lundin J, Haglund $\mathrm{C}$, von Smitten $\mathrm{K}$, von Boguslawsky $\mathrm{K}$, Nordling S: Ki-67, p53, Er-receptors, ploidy and S-phase as prognostic factors in $\mathrm{T} 1$ node negative breast cancer. Acta Oncol 1997, 36:369-374.

24. Railo M, Nordling S, von Boguslawsky K, Leivonen M, Kyllonen L, von Smitten K: Prognostic value of Ki-67 immunolabelling in primary operable breast cancer. $\mathrm{Br} J$ Cancer 1993, 68:579-583.

25. Veronese SM, Gambacorta M, Gottardi O, Scanzi F, Ferrari M, Lampertico $P$ : Proliferation index as a prognostic marker in breast cancer. Cancer 1993, 71:3926-3931.

26. Wintzer HO, Zipfel I, Schulte-Monting J, Hellerich U, von Kleist S: $\mathrm{Ki}-67$ immunostaining in human breast tumors and its relationship to prognosis. Cancer 1991, 67:421-428.

27. Archer SG, Eliopoulos A, Spandidos D, Barnes D, Ellis IO, Blamey RW, Nicholson RI, Robertson JF: Expression of ras p21, p53 and c-erbB-2 in advanced breast cancer and response to first line hormonal therapy. Br J Cancer 1995, 72:1 259-1266.

28. Daidone MG, Luisi A, Martelli G, Benini E, Veneroni S, Tomasic G, De Palo G, Silvestrini R: Biomarkers and outcome after tamoxifen treatment in node-positive breast cancers from elderly women. Br J Cancer 2000, 82:270-277.

29. Clahsen PC, van de Velde CJ, Duval C, Pallud C, Mandard AM, Delobelle-Deroide A, van den Broek L, Sahmoud TM, van de Vijver $\mathrm{MJ}$ : p53 protein accumulation and response to adjuvant chemotherapy in premenopausal women with node-negative early breast cancer. J Clin Oncol 1998, 16:470-479.

30. Bottini $A$, Berruti $A$, Bersiga $A$, Brizzi MP, Bruzzi $P$, Aguggini $S$ Brunelli A, Bolsi G, Allevi G, Generali D, et al:: Relationship between tumour shrinkage and reduction in Ki67 expression after primary chemotherapy in human breast cancer. $\mathrm{Br} J$ Cancer 2001, 85:1106-1112. 
31. Clarke RB, Laidlaw IJ, Jones LJ, Howell A, Anderson E: Effect of tamoxifen on Ki67 labelling index in human breast tumours and its relationship to oestrogen and progesterone receptor status. Br J Cancer 1993, 67:606-611.

32. MacGrogan G, Mauriac L, Durand M, Bonichon F, Trojani M, de Mascarel I, Coindre JM: Primary chemotherapy in breast invasive carcinoma: predictive value of the immunohistochemical detection of hormonal receptors, p53, c-erbB-2, MiB1, pS2 and GST pi. Br J Cancer 1996, 74:1458-1465.

33. Rudas M, Gnant MF, Mittlbock M, Neumayer R, Kummer A, Jakesz R, Reiner G, Reiner A: Thymidine labeling index and Ki-67 growth fraction in breast cancer: comparison and correlation with prognosis. Breast Cancer Res Treat 1994, 32:165-175.

34. Bottini A, Berruti A, Bersiga A, Brunelli A, Brizzi MP, Marco BD, Cirillo F, Bolsi G, Bertoli G, Alquati P, Dogliotti L: Effect of neoadjuvant chemotherapy on Ki67 labelling index, c-erbB-2 expression and steroid hormone receptor status in human breast tumours. Anticancer Res 1996, 16:3105-3110.

35. Ellis PA, Smith IE, Detre S, Burton SA, Salter J, A'Hern R, Walsh G, Johnston SR, Dowsett M: Reduced apoptosis and proliferation and increased $\mathrm{Bcl}-2$ in residual breast cancer following preoperative chemotherapy. Breast Cancer Res Treat 1998, 48:107-116.

36. Dardes RD, Horiguchi J, Jordan VC: A pilot study of the effects of short-term tamoxifen therapy on Ki-67 labelling index in women with primary breast cancer. Int $J$ Oncol 2000, 16:25-30.

37. Makris A, Powles TJ, Allred DC, Ashley S, Ormerod MG, Titley JC, Dowsett $\mathrm{M}$ : Changes in hormone receptors and proliferation markers in tamoxifen treated breast cancer patients and the relationship with response. Breast Cancer Res Treat 1998, 48:11-20.

38. Chang J, Powles TJ, Allred DC, Ashley SE, Clark GM, Makris A, Assersohn L, Gregory RK, Osborne CK, Dowsett M: Biologic markers as predictors of clinical outcome from systemic therapy for primary operable breast cancer. J Clin Oncol 1999, 17:3058-3063.

39. Billgren AM, Rutqvist LE, Tani E, Wilking N, Fornander T, Skoog L: Proliferating fraction during neoadjuvant chemotherapy of primary breast cancer in relation to objective local response and relapse-free survival. Acta Oncol 1999, 38:597-601.

40. Makris A, Powles TJ, Allred DC, Ashley SE, Trott PA, Ormerod MG, Titley JC, Dowsett M: Quantitative changes in cytological molecular markers during primary medical treatment of breast cancer: a pilot study. Breast Cancer Res Treat 1999, 53:51-59.

41. Honkoop AH, van Diest PJ, de Jong JS, Linn SC, Giaccone G, Hoekman K, Wagstaff J, Pinedo HM: Prognostic role of clinical, pathological and biological characteristics in patients with locally advanced breast cancer. Br J Cancer 1998, 77:621-626.

42. Bear HD, Anderson S, Brown A, Smith R, Mamounas EP, Fisher B, Margolese R, Theoret H, Soran A, Wickerham DL, et al:: The effect on tumor response of adding sequential preoperative docetaxel to preoperative doxorubicin and cyclophosphamide: preliminary results from National Surgical Adjuvant Breast and Bowel Project Protocol B-27. J Clin Oncol 2003, 21:4165-4174.

43. Buchholz TA, Davis DW, McConkey DJ, Symmans WF, Valero V, Jhingran A, Tucker SL, Pusztai L, Cristofanilli M, Esteva FJ, et al.: Chemotherapy-induced apoptosis and $\mathrm{Bcl}-2$ levels correlate with breast cancer response to chemotherapy. Cancer J 2003, 9:33-41.

44. Symmans WF, Volm MD, Shapiro RL, Perkins AB, Kim AY, Demaria S, Yee HT, McMullen H, Oratz R, Klein P, et al.: Paclitaxel-induced apoptosis and mitotic arrest assessed by serial fine-needle aspiration: implications for early prediction of breast cancer response to neoadjuvant treatment. Clin Cancer Res 2000, 6:4610-4617.

45. Chang J, Ormerod M, Powles TJ, Allred DC, Ashley SE, Dowsett $\mathrm{M}$ : Apoptosis and proliferation as predictors of chemotherapy response in patients with breast carcinoma. Cancer 2000, 89:2145-2152 